\title{
MicroRNA-200a-3p and GATA6 are abnormally expressed in patients with non-small cell lung cancer and exhibit high clinical diagnostic efficacy
}

\author{
JIE YU ${ }^{1 *}$, XINYUN HE ${ }^{1 *}$, CHUNJU FANG ${ }^{1}$, HAIXIA WU ${ }^{1}$, LEI HU ${ }^{2}$ and YINGBO XUE ${ }^{1}$ \\ ${ }^{1}$ Department of Oncology, Guizhou Provincial People's Hospital, Guiyang, Guizhou 550001; \\ ${ }^{2}$ Department of Laboratory Medicine, Guizhou Women's and Children's Hospital, \\ Guiyang, Guizhou 550003, P.R. China
}

Received March 19, 2021; Accepted November 16, 2021

DOI: $10.3892 /$ etm. 2022.11210

\begin{abstract}
Lung cancer is one of the main threats to human health. Survival of patients with lung cancer depends on timely detection and diagnosis. Among the genetic irregularities that control cancer development and progression, there are microRNAs (miRNAs/miRs). The present study aimed to investigate the expression patterns of miR-200a-3p and transcription factor GATA-6 (GATA6) in peripheral blood of patients with non-small cell lung cancer (NSCLC) and their clinical significance. The expression patterns of miR-200a-3p and GATA6 in the peripheral blood of patients with NSCLC and healthy subjects were measured via reverse transcription-quantitative PCR. The correlation between GATA6/miR-200a-3p expression and their diagnostic efficacy were analyzed by receiver operating characteristic curve analysis. The association between miR-200a-3p/GATA6 expression with the patient clinicopathological characteristics, and their correlation with carcinoembryonic antigen (CEA), neuron specific enolase (NSE) and squamous cell carcinoma antigen $(\mathrm{SCCAg})$ were evaluated. The cumulative survival rate was examined, and whether miR-200a-3p and GATA6 expression levels were independently correlated with the prognosis of NSCLC was analyzed using multivariate logistic regression model. The results demonstrated that the expression of miR-200a-3p was high and that of GATA6 was low in the peripheral blood of patients with NSCLC, and both exhibited high clinical diagnostic efficacy. miR-200a-3p was revealed
\end{abstract}

Correspondence to: Miss Yingbo Xue, Department of Oncology, Guizhou Provincial People's Hospital, 83 Zhongshan East Road, Nanming, Guiyang, Guizhou 550001, P.R. China

E-mail: xueyingbo1230@163.com

*Contributed equally

Key words: non-small cell lung cancer, microRNA-200a-3p, transcription factor GATA-6, clinical indicators, serum tumor markers, prognosis to target GATA6 by dual-luciferase assay. miR-200a-3p in the peripheral blood was correlated with TNM stage, lymph node metastasis and distal metastasis, while GATA6 in the peripheral blood was correlated with TNM stage and lymph node metastasis. miR-200a-3p and GATA6 were positively correlated with CEA and SCCAg, but not with NSE. High expression of miR-200a-3p and low expression of GATA6 predicted poor prognosis in patients with NSCLC. After adjusting for TNM stage, lymph node metastasis, distance metastasis, GATA6, CEA, NSE and SCCAg in the logistic regression model, it was indicated that the high expression of miR-200a-3p increased the risk of death in patients with NSCLC. Collectively, it was revealed that miR-200a-3p and GATA6 were abnormally expressed in the peripheral blood of patients with NSCLC. Serum levels of miR-200a-3p >1.475 and GATA6 <1.195 may assist the early diagnosis of NSCLC. GATA6 may function in NSCLC as a miR-200a-3p target, which may provide a future reference for NSCLC early diagnosis and treatment.

\section{Introduction}

A total of $\sim 85 \%$ of lung cancer cases are recognized as non-small cell lung cancer (NSCLC), which is a leading cause of human mortality worldwide with a critical 5-year survival (1) due to late diagnosis at advanced stages (2). Despite adequate treatment, which consists of thoracic radiotherapy (60-66 Gy in 30-33 daily fractions) with concomitant cisplatin-based chemotherapy (3), 50\% of medically inoperable patients with advanced NSCLC experience local recurrence; on the contrary, the avoidance of local recurrence in the early stage is up to $85-90 \%$ (4). Adequate treatment depends on accurate early diagnosis and effective treatment response, and peripheral blood detection of NSCLC can reduce the detection cost and avoid the potential damage caused by X-ray and pathological examination (5). Carcinoembryonic antigen (CEA) is a tumor marker, which represents the earliest tumor marker for the diagnosis of lung cancer (6). Squamous cell carcinoma antigen (SCCAg) is associated with the stage of lung cancer (7). Neuron specific enolase (NSE) is a specific enzyme distributed in neuroendocrine cells and involved 
in glycolysis (8). The clinical diagnostic value of the three genes in NSCLC has been largely discussed, but no single reliable index for the early diagnosis of NSCLC has been discovered (9). Molecular profiling of NSCLC in the past decade has revealed numerous oncogenic driving events (such as epidermal growth factor receptor mutation and anaplastic lymphoma kinase rearrangement) (10) in NSCLC, leading to several effective therapies, including tyrosine kinase inhibitors and immunotherapy (11). The present study aimed to unravel effective biomarkers in the peripheral blood of patients with NSCLC to achieve early diagnosis and timely intervention.

A number of studies have documented the involvement of microRNAs (miRNAs/miRs) in gene expression and evolution and the regulation of oncogene expression, which is closely associated with cell proliferation and differentiation and the occurrence, development and metastasis of malignant tumors $(12,13)$. In tumor tissues, changes in miRNA expression are tissue specific with high levels of stability and detectability, and miRNAs can act as oncogenes or tumor suppressors, which renders them novel targets for cancer diagnosis and treatment $(14,15)$. Essentially, the abnormal expression of the miR-200 family may promote the occurrence of NSCLC and may represent a potential diagnostic biomarker of NSCLC $(16,17)$. miR-200a-3p is highly expressed in NSCLC tissues (18). Although it has been demonstrated that miR-200a-3p is associated with NSCLC, the expression of miR-200a-3p in the peripheral blood of patients with NSCLC and its clinical diagnostic value have not been examined.

Transcription factor GATA-6 (GATA6) is a transcriptional regulator of the GATA family. As a cancer suppressor in several tumors, GATA6 is expressed at low level in patients with NSCLC (19). GATA6 is downregulated in lung adenocarcinoma (LUAD) and associated with LUAD metastasis and prognosis (20). In addition, the GATA6-PCAT1-Fyn-related kinase axis has been indicated to be a useful target for NSCLC (21). Moreover, miR-141 of the miR-200 family has been reported to regulate angiogenesis and affect cancer invasion and metastasis through multiple targets, including GATA6 (22). Therefore, we hypothesized that miR-200a-3p may serve a regulatory role in the occurrence and development of NSCLC by targeting GATA6. To the best of our knowledge, there is no report at present on the expression and clinical value of GATA6 in the peripheral blood of patients with NSCLC, and whether miR-200a-3p can serve a regulatory role in the occurrence and development of NSCLC by targeting GATA6. Therefore, the present study aimed to examine the expression and clinical significance of miR-200a-3p and GATA6 in the peripheral blood of patients with NSCLC and the possible regulatory mechanism between the two molecules, to provide a reference for the diagnosis, treatment and prognosis of NSCLC.

\section{Materials and methods}

Study subjects and grouping. In the current study, 60 patients pathologically diagnosed with NSCLC in Guizhou Provincial People's Hospital (Guiyang, China) between February 2014 and January 2015 were selected. The patients were free of mental illness, autoimmune diseases and other cancers. There were 42 males and 18 females with an age range of 39-80 years.
Inclusion criteria were as follows: i) According to the 2015 World Health Organization Lung Tumor Classification (23), the patients were initially diagnosed with NSCLC via histopathology; ii) none of the patients received lung cancer-related surgery or chemotherapy before enrollment; and iii) according to the TNM staging standard of the International Anti-Cancer Alliance (24), the subjects were allocated into stage I-II and III-IV. In addition, 60 individuals who underwent physical examination in Guizhou Provincial People's Hospital during the same period were included as the healthy control group, including 42 males and 18 females with an age range of 38-71 years. The clinicopathological data in the present study included age, sex, smoking, tumor size, histological types, TNM staging, lymph node metastasis (LNM) and distal metastasis. Written informed consent was obtained from each subject. The current study was approved by the Ethics Committee of Guizhou Provincial People's Hospital (Guiyang, China).

Sample collection. A total of $3 \mathrm{ml}$ venous blood was collected from fasting patients without chemotherapy or radiotherapy before operation and placed in a coagulation-promoting tube. After standing for $30 \mathrm{~min}$ at room temperature, the blood sample was centrifuged at $100 \mathrm{x}$ g for $10 \mathrm{~min}$ at room temperature. Then, the separated serum was stored at $-80^{\circ} \mathrm{C}$ until further use. The levels of CEA, NSE and SCCAg in the serum were determined using a Roche E601 electrochemiluminescence instrument [Roche Diagnostics (Shanghai) Co., Ltd.] using matching original reagents in strict accordance with the manufacturer's instructions. The normal reference range was $0-5.2 \mathrm{ng} / \mathrm{ml}$ for CEA, $0-16.3 \mathrm{ng} / \mathrm{ml}$ for NSE and $0-2.5 \mathrm{ng} / \mathrm{ml}$ for SCCAg.

Patient follow-up and records. Patients with NSCLC were followed up until August 2020. All patients were followed up every 6 months through clinic visits or telephone calls from the date of diagnosis. Each patient was followed up for at least four times. If the patients were not followed up, they were considered as lost cases and no longer recorded.

Reverse transcription quantitative polymerase chain reaction $(R T-q P C R)$. Total RNA from the serum samples was extracted using TRIzol ${ }^{\circledR}$ reagent (Invitrogen; Thermo Fisher Scientific, Inc.). According to the manufacturer's instructions, PrimeScript RT Reagent Kit (Takara Biotechnology Co., Ltd.) was employed to reverse transcribe total RNA into cDNA. According to the manufacturer's instructions, the cDNA products of miRNA were obtained via RT using a SuperScript ${ }^{\mathrm{TM}}$ IV One-Step RT-PCR System (Applied Biosystems; Thermo Fisher Scientific, Inc.). qPCR was performed on ABI 7900HT fast real-time PCR system (Applied Biosystems; Thermo Fisher Scientific, Inc.) using SYBR $^{\circledR}$ Premix Ex Taq ${ }^{\mathrm{TM}}$ II (Takara Biotechnology Co., Ltd.). The PCR procedure was as follows: Pre-denaturation at $95^{\circ} \mathrm{C}$ for $5 \mathrm{~min}$, followed by 40 cycles of denaturation at $95^{\circ} \mathrm{C}$ for $15 \mathrm{sec}$, annealing at $60^{\circ} \mathrm{C}$ for $20 \mathrm{sec}$ and extension at $72^{\circ} \mathrm{C}$ for $35 \mathrm{sec}$. GAPDH was used as the internal reference for GATA6 mRNA, and the expression of miR-200a-3p was normalized using cel-miR-39 (25-27). The data were analyzed based on the $2^{-\Delta \Delta \mathrm{Cq}}$ method $(28,29)$. The primer 
Table I. Primer sequences used for reverse transcription-quantitative PCR.

\begin{tabular}{lll}
\hline Gene & \multicolumn{1}{c}{ Forward (5'-3') } & \multicolumn{1}{c}{ Reverse (5'-3') } \\
\hline miR-200a-3p & TAACACTGTCTGGTAACGATGT & CATCTTACCGGACAGTGCTGGA \\
GATA6 & GTGCCAACTGTCACACCACA & GAGTCCACAAGCATTGCACAC \\
GAPDH & CTCAGACACCATGGGGAAGGTGA & ATGATCTTGAGGCTGTTGTCATA \\
cel-miR-39 & CGTATGAGCGTCACCGGGTGTAAATCA & CTCAAGTGTCGTGGAGTCGGCAA
\end{tabular}

miR, microRNA; GATA6, transcription factor GATA-6.

sequences of miR-200a-3p were selected according to a previous study (28). Primer sequences were synthesized by Sangon Biotech Co., Ltd., and are were presented in Table I.

Bioinformatics analysis. The pan-cancer analysis platform [The Encyclopedia of RNA Interactomes (ENCORI); http://starbase. sysu.edu.cn/panMirDiffExp.php] was used to analyze and predict the expression of miR-200a-3p and GATA6 in NSCLC (GSE48568, GPL570, PRJNA185966) (30-32). starBase v2.0 (http://starbase.sysu.edu.cn), miRDB (http://www.mirdb. org/) and TargetScan v7.1 (http://www.targetscan.org/) were employed to analyze and predict whether there was a target binding relationship between miR-200a-3p and GATA6.

Dual-luciferase reporter gene assay and cell transfection. The binding sites of miR-200a-3p and GATA6 were predicted via bioinformatics analysis as aforementioned. The complementary wild-type (WT) binding sequence and the mutant (MUT) sequence of the 3'-untranslated region of GATA6 were amplified and cloned into pmiRGLO vector (Promega Corporation) to obtain pmiRGLO-GATA6-WT and pmiRGLO-GATA6-MUT plasmids (Fig. S1). The WT sequence was randomly scrambled and amplified to obtain the MUT sequence. The constructed vectors were mixed with $60 \mathrm{nM}$ mimic negative control (NC) or $60 \mathrm{nM}$ miR-200a-3p mimic (Shanghai GenePharma Co., Ltd.) and co-transfected with Lipofectamine ${ }^{\circledR} 2000$ (Invitrogen; Thermo Fisher Scientific, Inc.) into $293 \mathrm{~T}$ cells (Shanghai Institute of Biochemistry and Cell Biology) cultured to $80 \%$ confluence at $37^{\circ} \mathrm{C}$. With Renilla luciferase as the internal reference, the luciferase activities were detected using Dual-Luciferase Assay kit (cat. no. ZY130595; Shanghai Zeye Biotechnology Co., Ltd., Shanghai, China) after $48 \mathrm{~h}$ according to the manufacturer's instructions. 293T cells were cultured in high-glucose DMEM (cat. no. SNM-002A; Sunncell Wuhan) containing 10\% FBS (Gibco; Thermo Fisher Scientific, Inc.) and 1\% penicillin/streptomycin at $37^{\circ} \mathrm{C}$ containing $5 \% \mathrm{CO}_{2}$.

In addition, the human NSCLC cell line NCI-H1437 (Shanghai Huiying biological technology Co., Ltd.) was transfected with $60 \mathrm{nM}$ mimic NC or $60 \mathrm{nM}$ miR-200a-3p mimic and $120 \mathrm{nM}$ inhibitor NC or $120 \mathrm{nM}$ miR-200a-3p inhibitor at $37^{\circ} \mathrm{C}$ using Lipofectamine ${ }^{\circledR} 2000$ (Invitrogen; Thermo Fisher Scientific, Inc.). After $48 \mathrm{~h}$, the expression of GATA6 in each group was detected via RT-qPCR. NCI-H1437 cells were cultured in RPMI-1640 medium (Procell Life Science \& Technology Co., Ltd.) with $10 \% \mathrm{FBS}$ at $37^{\circ} \mathrm{C}$ and sub-cultured at a $1: 4$ ratio.
miR-200a-3p mimic was the simulant of miR-200a-3p, and its sequence was 5'-UAACACUGUCUGGUAACGAUGU-3', while the sequence of mimic NC was 5'-CUGUUACACGCA GUUGAUGUAA-3'. miR-200a-3p inhibitor was a chemically modified complementary single chain of mature miR-200a-3p (cat. no. miR20000682-1-5), and inhibitor NC was its negative control (cat. no. miR02102-1-1), which were designed and synthesized by Guangzhou RiboBio Co., Ltd.

Statistical analysis. SPSS 21.0 (IBM Corp.) and GraphPad Prism 8.01 (GraphPad Software, Inc.) were used for statistical analysis and plotting of the data. The normal distribution of the continuous variables was verified using Kolmogorov-Smirnov test. Numerical data are presented as the mean \pm standard deviation and categorical data as count and percentage. An unpaired Student's t-test was employed for the analysis between two groups and one-way ANOVA was used for the comparison among multiple groups (continuous variables). Tukey's multiple comparisons test was utilized after one-way ANOVA. The $\chi^{2}$ test was employed for the analysis of categorical variables. Pearson correlation coefficient method was applied to analyze correlations. The $r$ value was the correlation coefficient between variables in the sample. When $r=0$, it indicates no correlation; when $\mathrm{r} \leq 0.3$, it indicates poor correlation; when $0.3<\mathrm{r} \leq 0.8$, it indicates medium correlation; when $\mathrm{r}>0.8$, it indicates high correlation. The cut-off point, sensitivity, specificity and the area under the curve (AUC) of the receiver operating characteristic (ROC) curves were obtained using MedCalc Statistical Software version 14.8.1 (MedCalc Software bvba). A multivariate logistic regression model was used to analyze whether the expression of miR-200a-3p and GATA6 was independently associated with the prognosis of NSCLC. The Cox proportional hazard regression model and the Kaplan-Meier method were used to evaluate the overall survival (OS), and the log-rank test was used to create the survival curve. $\mathrm{P}<0.05$ was considered to indicate a statistically significant difference.

\section{Results}

miR-200a-3p is expressed at a high and GATA6 at a low level in the peripheral blood of patients with NSCLC. To examine the diagnostic value of miR-200a-3p and GATA6 for NSCLC, the pan-cancer analysis platform ENCORI was used to firstly predict the expression of miR-200a-3p and GATA6 in NSCLC. Lung squamous cell carcinoma (LUSC) and LUAD account for $\sim 90 \%$ of NSCLC (33), therefore these groups were further 
A

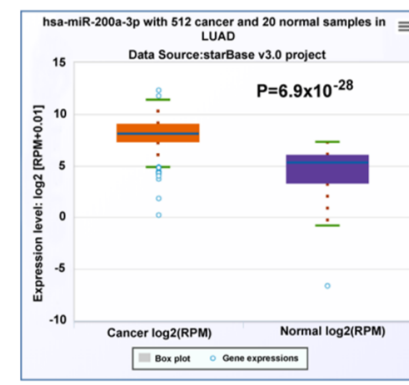

C

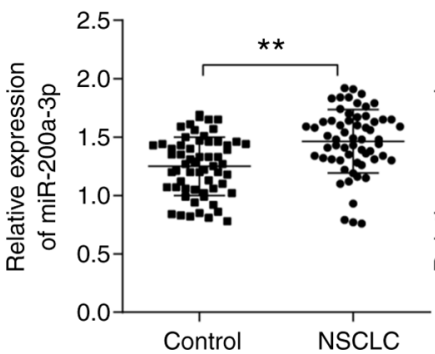

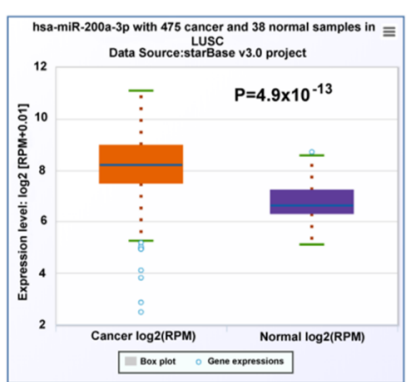

D

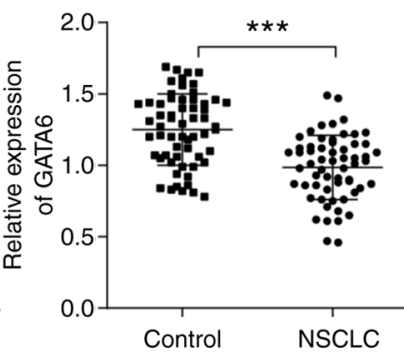

B

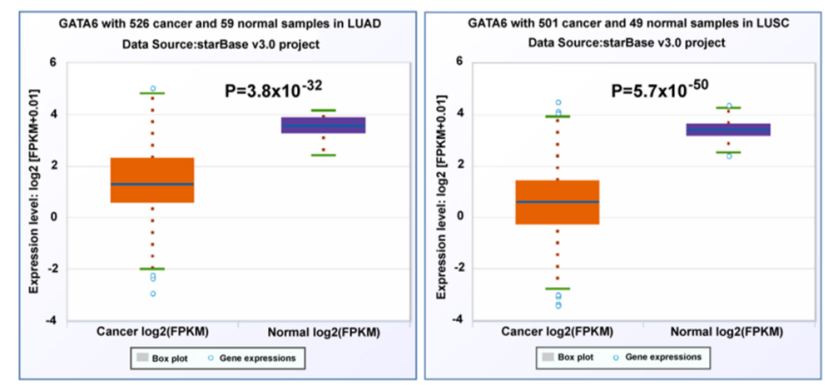

E

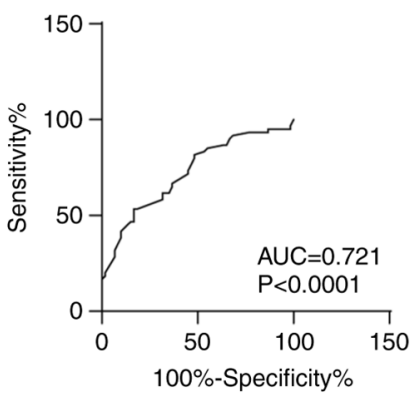

F

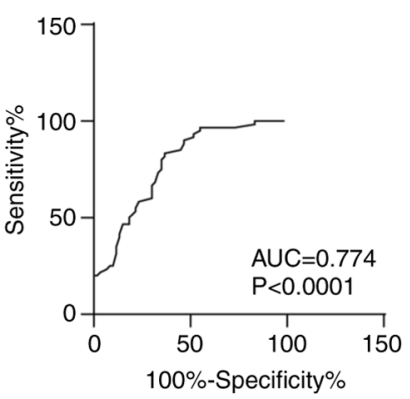

Figure 1. miR-200a-3p and GATA6 exhibit high clinical diagnostic efficacy for patients with NSCLC. (A) Expression of miR-200a-3p in LUSC and LUAD was analyzed using the ENCORI pan-cancer analysis database. (B) Expression of GATA6 in LUSC and LUAD was analyzed using the ENCORI pan-cancer analysis database. The expression of (C) miR-200a-3p and (D) GATA6 in the peripheral blood of patients with NSCLC and healthy subjects was detected via reverse transcription-quantitative PCR. The diagnostic efficacy of (E) miR-200a-3p and (F) GATA6 in the peripheral blood of patients with NSCLC was analyzed using ROC curve. ${ }^{* *} \mathrm{P}<0.01,{ }^{* * * *} \mathrm{P}<0.001$. The data in panels $\mathrm{C}$ and D were analyzed via an unpaired Student's t-test. miR, microRNA; GATA6, transcription factor GATA-6; LUSC, lung squamous cell carcinoma; LUAD, lung adenocarcinoma; NSCLC, non-small cell lung cancer; AUC, area under the receiver operating characteristic curve; RPM, reads per million mapped reads; FKPM, fragments per kilobase of transcript per million reads mapped.

explored. The results demonstrated that compared with the normal group, miR-200a-3p was significantly elevated in LUSC and LUAD (Fig. 1A), whereas GATA6 was significantly decreased in LUSC and LUAD (Fig. 1B).

Therefore, the expression of miR-200a-3p and GATA6 in the peripheral blood of patients with NSCLC and healthy controls was detected using RT-qPCR. The results revealed that miR-200a-3p in the peripheral blood of patients with NSCLC was remarkably higher than that of healthy controls (Fig. 1C), and GATA6 was lower than that of healthy controls (Fig. 1D).

Subsequently, the ROC curve was used to analyze the diagnostic value of miR-200a-3p and GATA6 in NSCLC. The AUC of miR-200a-3p in the peripheral blood of patients with NSCLC was 0.721 , with a cut-off value was 1.475 , a sensitivity of $83.33 \%$ and a specificity of $63.33 \%$ (Fig. 1E). The AUC of GATA6 in the peripheral blood of patients with NSCLC was 0.774 , with a cut-off value of 1.195 , a sensitivity of $53.33 \%$ and a specificity of $83.33 \%$ (Fig. 1F). The results revealed that cut-off values of miR-200a-3p $>1.475$ and GATA6 $<1.195$ could assist in the early diagnosis of NSCLC.

GATA6 is the target of miR-200a-3p. Bioinformatics analysis was used to analyze and predict whether there was a targeted binding relationship between miR-200a-3p and GATA6. starBase, miRDB (score $>70$ ) and TargetScan (preferentially conserved targeting $>80$ ) were used to analyze and predict the downstream targets of miR-200a-3p, and 22 downstream target genes of miR-200a-3p were demonstrated to be common in all three databases (Table SI and Fig. 2A). Among these genes,
GATA6 was indicated to be downregulated in NSCLC tissues compared with normal tissues, which may be used as a useful target for the intervention of NSCLC. In addition, miR-141 of the miR-200 family can regulate angiogenesis via multiple targets, including GATA6, thus affecting cancer invasion and metastasis $(19,21,22)$. Therefore, GATA6 was selected for further study.

Subsequently, the correlation between the expression of miR-200a-3p and GATA6 in the peripheral blood of patients with NSCLC was analyzed, and a negative correlation was observed between them ( $r=-0.634$; Fig. 2B). Next, the targeted binding relationship between miR-200a-3p and GATA6 was verified via dual-luciferase assay. Compared with the cells co-transfected with pmiRGLO-GATA6-WT plasmid and mimic NC, the luciferase enzyme activity of 293 T cells co-transfected with pmiRGLO-GATA6-WT and miR-200a-3p was significantly decreased, while this difference was not observed between cells transfected with pmiRGLO-GATA6-MUT and miR-200a-3p or mimic NC (Fig. 2C). In addition, miR-200a-3p was overexpressed or knocked down by transfecting miR-200a-3p mimic or miR-200a-3p inhibitor into the NSCLC cell line NCI-H1437. The mRNA level of GATA6 was detected via RT-qPCR. Overexpression of miR-200a-3p significantly decreased, while knockdown of miR-200a-3p significantly increased the mRNA level of GATA6 (Fig. 2D). These results suggested that GATA6 is a target gene of miR-200a-3p.

Association of miR-200a-3p and GATA6 with clinical indicators. To further analyze the clinical significance of 
A

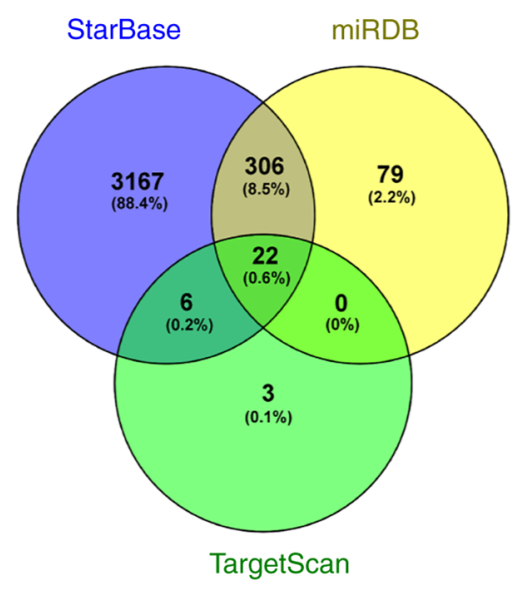

C

Binding site of hsa-miR-200a-3p on GATA6:

Show $10 \quad \checkmark$ entries
B

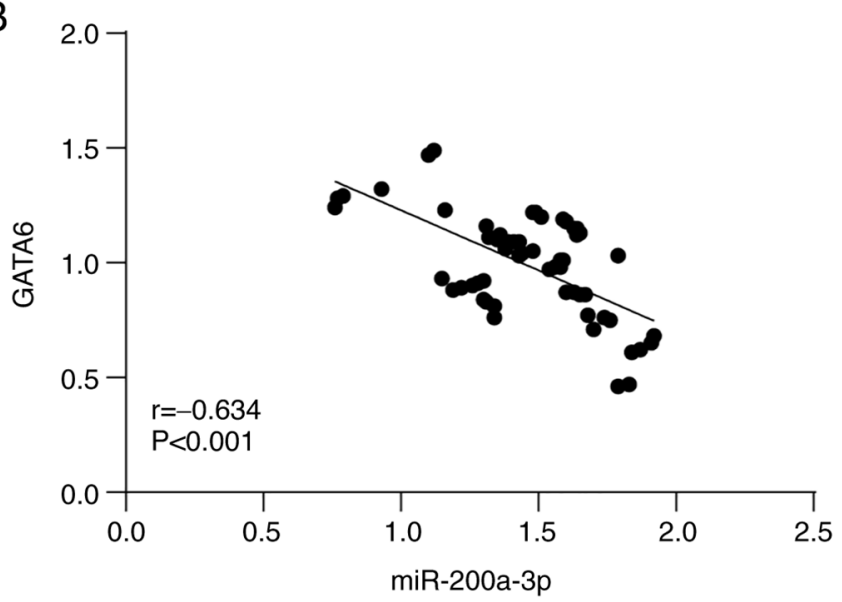

\begin{tabular}{|c|c|c|c|}
\hline BindingSite & $\uparrow$ & Class: & Alignment \\
\hline chr18:19781462-19781468[+] & $\uparrow$ & 8 mert & 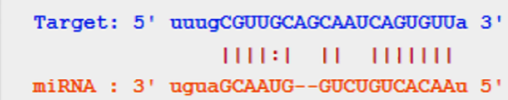 \\
\hline
\end{tabular}

D

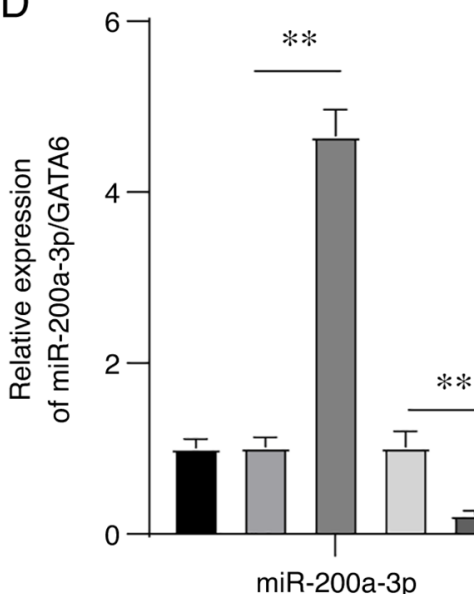

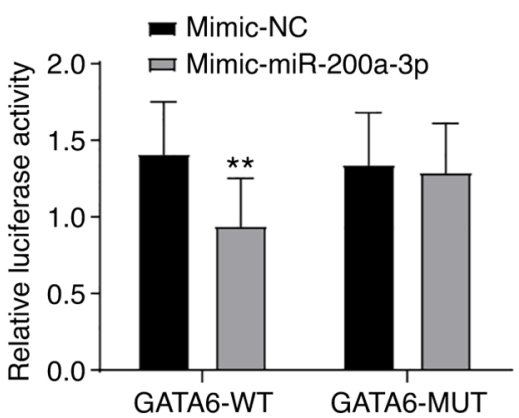

- NCl-H1437

$\square$ Mimic NC

ఐ miR-200a-3p mimic

$\square$ Inhibitor NC

ए miR-200a-3p inhibitor

Figure 2. GATA6 is a target gene of miR-200a-3p. (A) starBase, miRDB and TargetScan were used to predict the downstream target genes of miR-200a-3p and the common genes between the three databases were obtained. (B) Correlation analysis of miR-200a-3p and GATA6 expression in the peripheral blood of patients with non-small cell lung cancer. (C) Dual-luciferase assay was used to verify the targeted binding of miR-200a-3p and GATA6. (D) The expression of miR-200a-3p and GATA6 mRNA were detected via reverse transcription-quantitative PCR. Unpaired Student's t-test was used for data analysis in panel C, while the data in panels D were analyzed by one-way ANOVA followed by Tukey's multiple comparisons test. ${ }^{* *} \mathrm{P}<0.01$ vs. the indicated groups or mimic-NC. miR/miRNA, microRNA; GATA6, transcription factor GATA-6; NC, negative control; WT, wild-type; MUT, mutant.

miR-200a-3p and GATA6 in the peripheral blood of patients with NSCLC, the patients were assigned into miR-200a-3p high expression and miR-200a-3p low expression group according to the median expression of miR-200a-3p in the peripheral blood (median, 1.48; range, 0.76-1.92). At the same time, the patients were classified into GATA6 high expression and GATA6 low expression group according to the median expression of GATA6 expression in the peripheral blood (median, 1.02; range, 0.46-1.49). The clinicopathological features of the two groups were analyzed (Table II). The results demonstrated that miR-200a-3p and GATA6 in the peripheral blood were not associated with age, sex, smoking history, histological type, tumor size and distant metastasis, but were significantly associated with TNM stage and LNM.

Subsequently, the expression of miR-200a-3p and GATA6 in patients with NSCLC at different stages was further explored. Compared with healthy controls, miR-200a-3p expression in the peripheral blood of patients with NSCLC at stage I-II and III-IV was significantly increased (Fig. 3A), and that of GATA6 was significantly decreased (Fig. 3B). In addition, compared with stage I-II, miR-200a-3p expression in the peripheral blood of patients with NSCLC at stage III-IV was significantly increased, while that of GATA6 was significantly decreased. Therefore, it was speculated that the expression of 
Table II. Association between the expression of miR-200a-3p and GATA6 with clinical indicators.

A, miR-200a-3p

Expression

\begin{tabular}{|c|c|c|c|c|}
\hline Clinical data & Patient number $(n=60)$ & High, n (\%) & Low, n (\%) & $\mathrm{P}$-value \\
\hline \multicolumn{5}{|l|}{ Sex } \\
\hline Male & 42 & $22(52.38)$ & $20(47.62)$ & \\
\hline Female & 18 & $8(44.44)$ & $10(52.38)$ & 0.573 \\
\hline \multicolumn{5}{|l|}{ Age, years } \\
\hline$\leq 60$ & 29 & $15(51.72)$ & $14(48.28)$ & \\
\hline$>60$ & 31 & $15(48.39)$ & $16(51.65)$ & 0.796 \\
\hline \multicolumn{5}{|c|}{ Smoking history } \\
\hline Yes & 26 & $11(42.31)$ & $15(57.69)$ & \\
\hline No & 34 & $19(55.88)$ & $15(44.12)$ & 0.297 \\
\hline \multicolumn{5}{|c|}{ Histological types } \\
\hline LUSC & 35 & $19(54.29)$ & $16(45.71)$ & \\
\hline LUAD & 25 & $11(44.00)$ & $14(56.00)$ & 0.432 \\
\hline \multicolumn{5}{|c|}{ Tumor size, $\mathrm{cm}$} \\
\hline$\leq 3$ & 24 & $11(45.83)$ & $13(54.17)$ & \\
\hline$>3$ & 36 & $19(52.78)$ & $17(47.22)$ & 0.299 \\
\hline \multicolumn{5}{|l|}{ TNM stage } \\
\hline I-II & 42 & $16(38.10)$ & $26(61.90)$ & \\
\hline III-IV & 18 & $14(77.78)$ & $4(22.22)$ & 0.002 \\
\hline \multicolumn{5}{|l|}{ LNM } \\
\hline Yes & 22 & $15(68.18)$ & $7(31.82)$ & \\
\hline No & 38 & $15(39.47)$ & $23(60.53)$ & 0.016 \\
\hline \multicolumn{5}{|c|}{ Distant metastasis } \\
\hline Yes & 32 & $20(62.50)$ & $12(37.50)$ & \\
\hline No & 28 & $10(35.71)$ & $18(64.29)$ & 0.019 \\
\hline
\end{tabular}

B, GATA6

Expression

\begin{tabular}{|c|c|c|c|c|}
\hline Clinical data & Patient number $(n=60)$ & High, n (\%) & Low, n (\%) & P-value \\
\hline \multicolumn{5}{|l|}{ Sex } \\
\hline Male & 42 & $19(45.24)$ & $23(54.76)$ & \\
\hline Female & 18 & $11(61.11)$ & $7(38.89)$ & 0.130 \\
\hline \multicolumn{5}{|l|}{ Age, years } \\
\hline$\leq 60$ & 29 & $16(55.17)$ & $13(44.83)$ & \\
\hline$>60$ & 31 & $14(45.16)$ & $17(54.84)$ & 0.219 \\
\hline \multicolumn{5}{|c|}{ Smoking history } \\
\hline Yes & 26 & $14(53.85)$ & $12(46.15)$ & \\
\hline No & 34 & $16(47.06)$ & $18(52.94)$ & 0.301 \\
\hline \multicolumn{5}{|c|}{ Histological types } \\
\hline LUSC & 35 & 17 (48.57) & $18(51.43)$ & \\
\hline LUAD & 25 & $13(52.00)$ & $12(48.00)$ & 0.397 \\
\hline \multicolumn{5}{|l|}{ Tumor size, cm } \\
\hline$\leq 3$ & 24 & $11(45.83)$ & $13(54.17)$ & \\
\hline$>3$ & 36 & $19(52.78)$ & $17(47.22)$ & 0.299 \\
\hline \multicolumn{5}{|l|}{ TNM stage } \\
\hline I-II & 42 & $25(59.52)$ & $17(40.48)$ & \\
\hline III-IV & 18 & $5(27.78)$ & $13(72.22)$ & 0.012 \\
\hline
\end{tabular}


Table II. Continued.

\section{B, GATA6}

\begin{tabular}{|c|c|c|c|c|}
\hline \multirow[b]{2}{*}{ Clinical data } & \multirow[b]{2}{*}{ Patient number $(n=60)$} & \multicolumn{2}{|c|}{ Expression } & \multirow[b]{2}{*}{ P-value } \\
\hline & & High, n (\%) & Low, n (\%) & \\
\hline \multicolumn{5}{|l|}{ LNM } \\
\hline Yes & 22 & $6(27.27)$ & $16(72.73)$ & \\
\hline No & 38 & $24(63.16)$ & $14(36.84)$ & 0.004 \\
\hline \multicolumn{5}{|c|}{ Distant metastasis } \\
\hline Yes & 32 & $13(40.63)$ & $19(59.38)$ & \\
\hline No & 28 & $17(56.67)$ & $11(36.67)$ & 0.060 \\
\hline
\end{tabular}

miR, microRNA; GATA6, transcription factor GATA-6; LNM, lymph node metastasis; LUSC, lung squamous cell carcinoma; LUAD, lung adenocarcinoma.
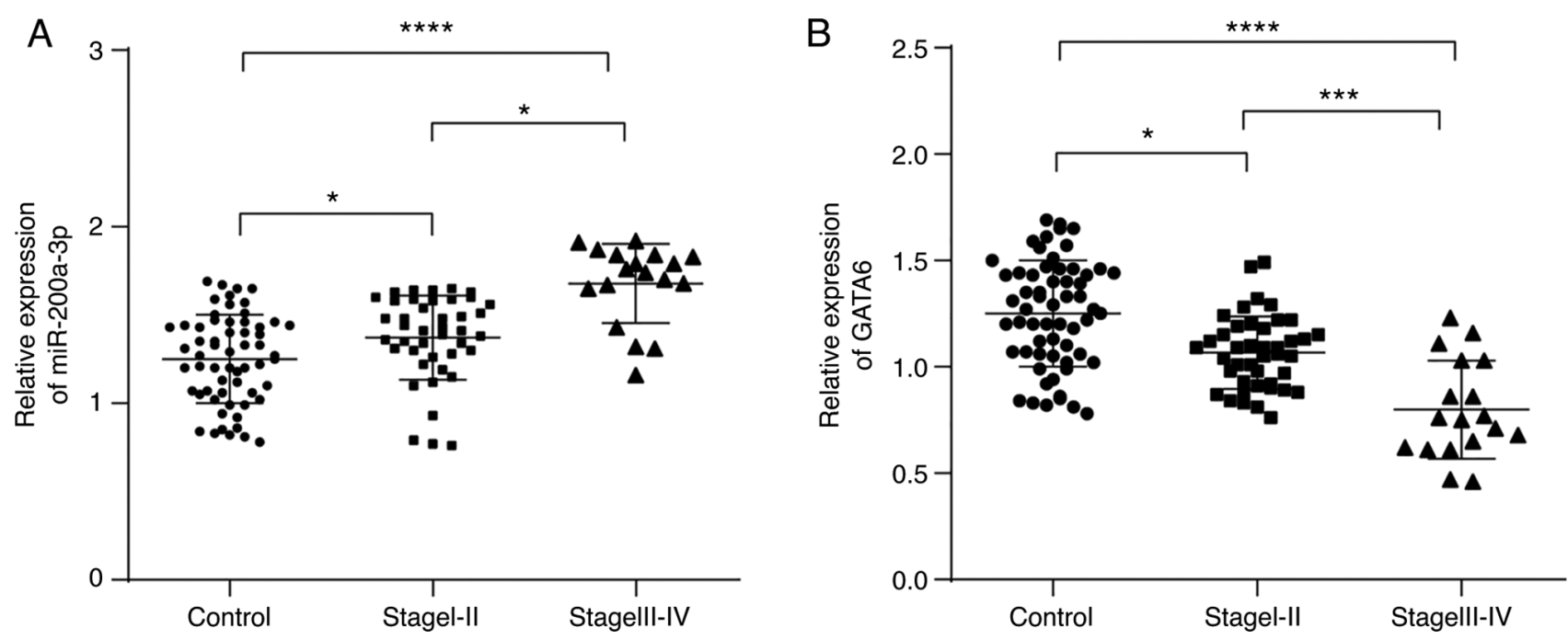

Figure 3. miR-200a-3p and GATA6 may be associated with the clinical progression of NSCLC. (A) miR-200a-3p and (B) GATA6 expression in the peripheral blood of patients with NSCLC at different stages and healthy subjects. The data were analyzed via one-way ANOVA and Tukey's multiple comparisons test. ${ }^{*} \mathrm{P}<0.05,{ }^{* * *} \mathrm{P}<0.001,{ }^{* * * * *} \mathrm{P}<0.0001$. miR, microRNA; GATA6, transcription factor GATA-6; NSCLC, non-small cell lung cancer.

miR-200a-3p and GATA6 may be associated with the clinical progression of NSCLC.

Correlation of miR-200a-3p and GATA6 with serum tumor markers. In addition, the serum tumor marker CEA, NSE and SCCAg levels in the peripheral blood of patients with NSCLC were detected [CEA $(21.0 \pm 6.9 \mathrm{ng} / \mathrm{ml})$, NSE $(23.6 \pm 9.7 \mathrm{ng} / \mathrm{ml})$, SCCAg $(4.6 \pm 2.8 \mathrm{ng} / \mathrm{ml})]$, and their correlation with the levels of miR-200a-3p and GATA6 was analyzed (Fig. 4A-F). The results demonstrated that miR-200a-3p and GATA6 were positively correlated with CEA and SCCAg, but not with NSE.

High expression of miR-200a-3p and low expression of GATA6 predicts poor prognosis in patients with NSCLC. Finally, the prognostic significance of miR-200a-3p and GATA6 expression in the peripheral blood of patients with NSCLC was analyzed. The patients were followed up regularly every 6 months. Among them, 31 patients died or were lost during the follow-up, including 11 cases in miR-200a-3p low expression group, 20 cases in
miR-200a-3p high expression group, 19 cases in GATA6 low expression group and 12 cases in GATA6 high expression group. Furthermore, the cumulative survival rates of the two groups of patients with NSCLC were compared and analyzed. The results demonstrated that the cumulative survival rate of the miR-200a-3p high expression group was markedly lower than that of the miR-200a-3p low expression group (Fig. 5A). The cumulative survival rate of the GATA6 low expression group was notably lower than that of the GATA6 high expression group (Fig. 5B). In addition, the expressions of miR-200a-3p and GATA6 in the peripheral blood were further compared and analyzed using the ROC curve to predict the adverse prognosis of NSCLC. The AUC of miR-200a-3p for NSCLC adverse prognosis was 0.653 , with a sensitivity of $64.52 \%$ and a specificity of $68.97 \%$. The AUC of GATA6 for NSCLC adverse prognosis was 0.616 , with a sensitivity of $48.39 \%$ and a specificity of $75.86 \%$. There were no significant differences between them (Fig. 5C). It was suggested that high expression of miR-200a-3p and low expression of GATA6 predicted poor prognosis. 
A
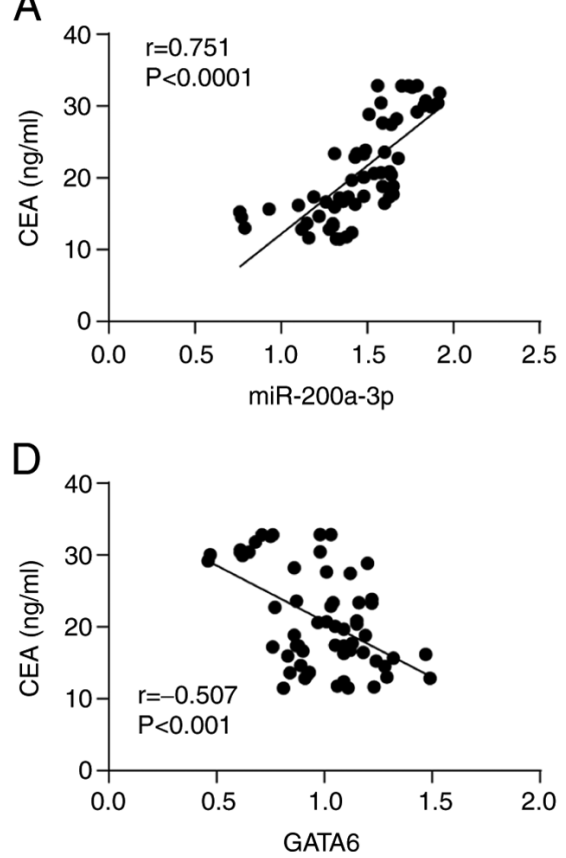

$\mathrm{B}$

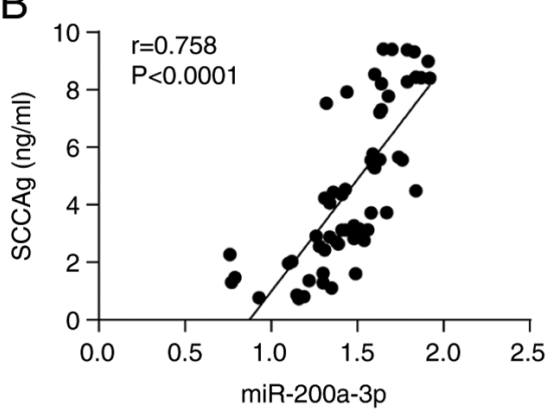

$\mathrm{E}$

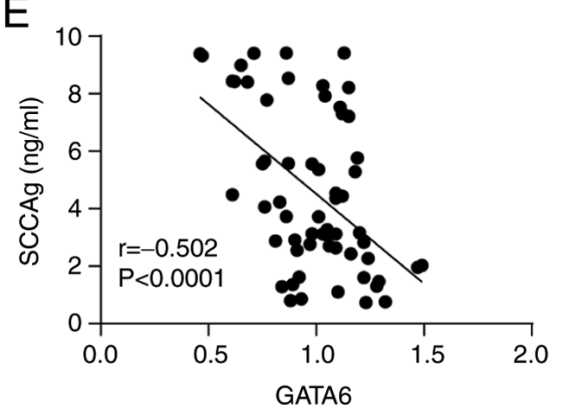

C

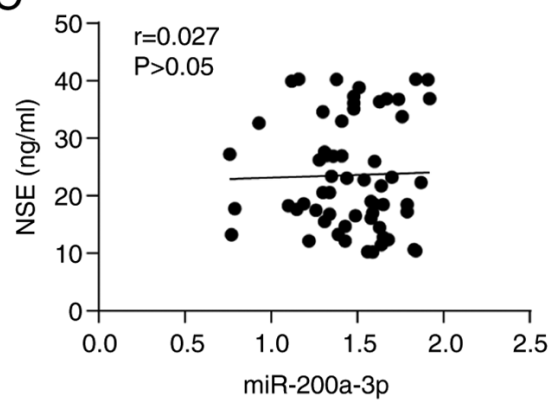

$\mathrm{F}$

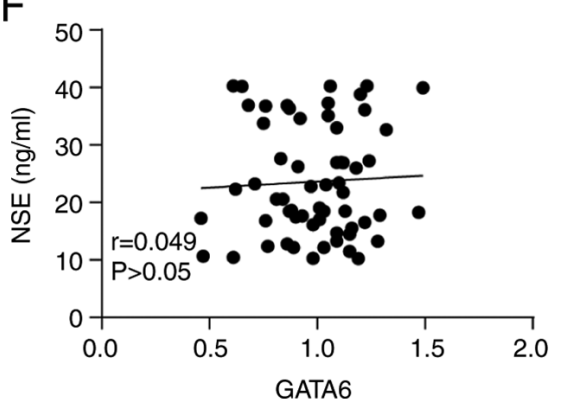

Figure 4. miR-200a-3p and GATA6 are correlated with the serum tumor markers CEA and SCCAg. Correlation analysis of miR-200a-3p with (A) CEA, (B) SCCAg and (C) NSE levels in the peripheral blood via Pearson analysis. Correlation analysis of GATA6 with (D) CEA, (E) SCCAg and (F) NSE levels in the peripheral blood via Pearson analysis. miR, microRNA; GATA6, transcription factor GATA-6; CEA, carcinoembryonic antigen; NSE, neuron specific enolase; SCCAg, squamous cell carcinoma antigen.

A

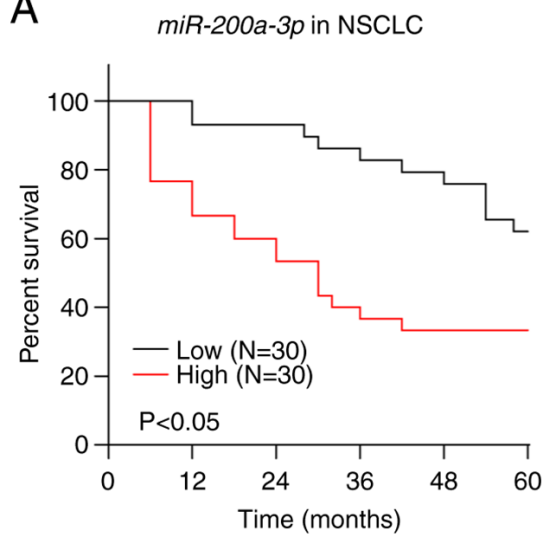

B

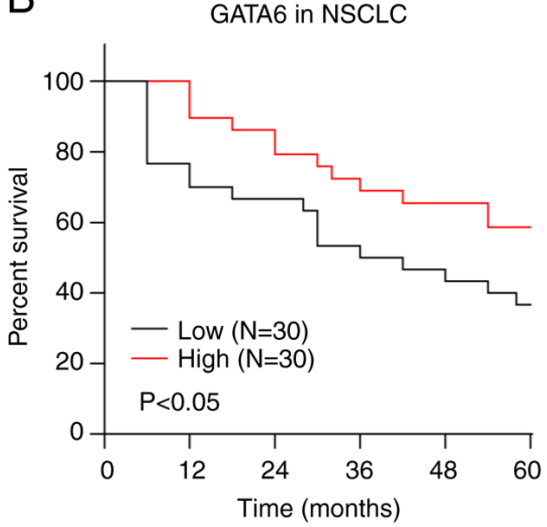

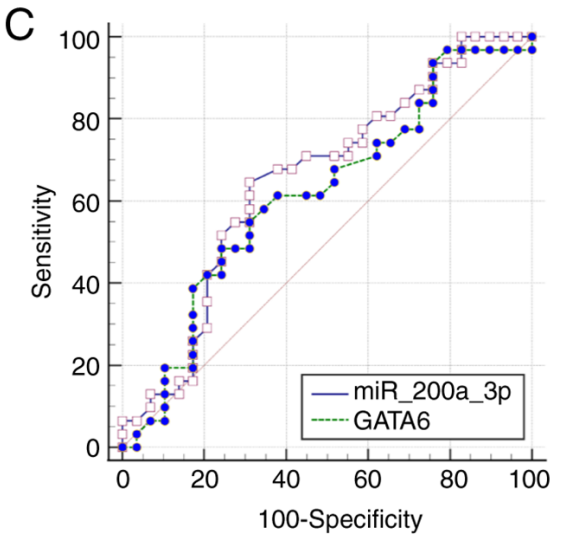

Figure 5. High expression of miR-200a-3p and low expression of GATA6 predict poor prognosis in patients with NSCLC. Prognostic analysis of (A) miR-200a-3p and (B) GATA6 for NSCLC. Kaplan-Meier analysis demonstrated the cumulative survival rate, and the log-rank test determined the difference of cumulative survival rate between groups. (C) The prognostic value of miR-200a-3p and GATA6 was compared and analyzed using the ROC curve. miR, microRNA; GATA6, transcription factor GATA-6; NSCLC, non-small cell lung cancer.

Furthermore, to further analyze whether there was an independent association between the expression of miR-200a-3p and GATA6 and the prognosis of NSCLC, the indexes with $\mathrm{P}<0.1$ from the clinicopathological associations presented in Table II and the serum tumor markers CEA and SCCAg that were significantly correlated with miR-200a-3p and GATA6 were included in a multivariate logistic regression model. After adjusting for TNM stage, lymph node metastasis, distant metastasis, GATA6, CEA, NSE and SCCAg in the logistic regression model, it was indicated that high expression of miR-200a-3p significantly increased the risk of death in patients with NSCLC (OR, 17.917; 95\% CI, 1.822-176.157; Table III), indicating that the expression of miR-200a-3p was independently associated with poor prognosis in NSCLC.

\section{Discussion}

In general, miRNAs are stably expressed in the blood and determining their expression levels is relatively simple (34). A previous study explored the association between the levels of certain peripheral blood markers, such as absolute neutrophil count, lymphocyte count, monocyte count and eosinophil count, as well as serum C-reactive protein and lactate dehydrogenase levels, and immune-related adverse events, OS and progression-free survival in NSCLC $(35,36)$. The present 
Table III. Multivariate logistic regression analysis of prognostic indicators for non-small cell lung cancer.

\begin{tabular}{lrrl}
\hline Variable & P-value & \multicolumn{1}{c}{ OR } & \multicolumn{1}{c}{$95 \%$ CI } \\
\hline TNM stage & 0.115 & 0.220 & $0.034-1.447$ \\
Lymph node metastasis & 0.119 & 4.583 & $0.676-31.071$ \\
Distant metastasis & 0.377 & 0.495 & $0.104-2.354$ \\
miR-200a-3p & 0.013 & 17.917 & $1.822-176.157$ \\
GATA6 & 0.230 & 0.039 & $0.000-7.801$ \\
CEA & 0.111 & 0.888 & $0.768-1.028$ \\
SCCAg & 0.139 & 0.754 & $0.518-1.096$ \\
NSE & 0.261 & 0.960 & $0.894-1.031$ \\
\hline
\end{tabular}

miR, microRNA; CEA, carcinoembryonic antigen; NSE, neuron specific enolase; SCCAg, squamous cell carcinoma antigen; GATA6, transcription factor GATA-6; OR, odds ratio.

study explored the expression and clinical significance of miR-200a-3p and GATA6, and demonstrated that the abnormal expression of miR-200a-3p/GATA6 in the peripheral blood of patients with NSCLC has a high clinical diagnostic efficiency and prognostic value.

miRNAs represent molecular biomarkers and potential targets for therapeutic interventions in NSCLC, especially the miR-200 family members (16,37). The pan-cancer analysis platform ENCORI used in the current study demonstrated that miR-200a-3p was elevated, whereas GATA6 was significantly decreased in LUSC and LUAD, which was also confirmed in the peripheral blood of clinical patients with NSCLC. The crucial and complex roles of the miR-200 family have been discussed in lung cancer (16). miR-200a-3p is upregulated in lung cancer and the subtype LUSC (38-40). GATA6 is a potent and clinically relevant tumor suppressor gene in LUAD (41). Disease-related miRNAs have been extensively studied, and several blood-based miRNA tests have been developed for lung cancer diagnosis, with reasonable sensitivity and specificity (42-44). Subsequently, the ROC curve method was used to analyze the diagnostic value of miR-200a-3p and GATA6. The AUC of miR-200a-3p in the peripheral blood of patients with NSCLC was 0.721 , and the cut-off value was 1.475 with a sensitivity of $83.33 \%$ and a specificity of $63.33 \%$. The AUC of GATA6 in the peripheral blood of patients with NSCLC was 0.774 , and the cut-off value was 1.195 with a sensitivity of $53.33 \%$ and a specificity of $83.33 \%$. To sum up, miR-200a-3p $>1.475$ and GATA6 <1.195 may assist in the early diagnosis of NSCLC.

Through database prediction and dual-luciferase assay, it was validated that GATA6 is a target gene of miR-200a-3p. miR-200a-3p upregulation has been indicated to independently lower TNF- $\alpha$-induced expression of transcription factor GATA6 (45). Next, the clinical significance of miR-200a-3p and GATA6 were analyzed. The patients were allocated into miR-200a-3p and GATA6 high/low expression groups according to the median expression of miR-200a-3p and GATA6. The analysis of clinicopathological features demonstrated that the levels of miR-200a-3p and GATA6 in the peripheral blood were not associated with age, sex, smoking history, histological type and tumor size, but were significantly associated with TNM stage, LNM and distal metastasis (not for GATA6). Consistently, another study has revealed that miR-200c overexpression is significantly associated with LNM, advanced TNM and poor survival rates in NSCLC (46). Higher level of GATA6 in LUAD was significantly associated with longer survival of patients in all stages (41). Furthermore, compared with stage I-II, the expression of miR-200a-3p in the peripheral blood of patients with NSCLC at stage III-IV was increased while that of GATA6 was significantly decreased. Similarly, miR-200a has been associated with cancer metastasis (47). Therefore, we hypothesized that miR-200a-3p and GATA6 expression may be associated with the clinical progression of NSCLC.

In the treatment process of NSCLC, blood-based biomarkers can help guide treatment decisions by monitoring tumor load, minimal residual or recurrent disease and genetic alterations (48). CEA, SCCAg and NSE are typical serum tumor markers $(6,8,49)$ and exhibit high clinical reliability in NSCLC. Therefore, we evaluated their expression and correlation with miR-200a-3p and GATA6. The results demonstrated that miR-200a-3p and GATA6 were correlated with CEA and SCCAg, but not with NSE. There is no report on the correlation of the three biomarkers with miR-200a-3p and GATA6 in the peripheral blood of patients with NSCLC, to the best of our knowledge, therefore the present study demonstrated this analysis for the first time. Finally, the prognostic significance of miR-200a-3p and GATA6 was analyzed. The cumulative survival rate of the miR-200a-3p high expression group was markedly lower than that of the miR-200a-3p low expression group, and the cumulative survival rate of the GATA6 low expression group was notably lower than that of GATA6 high expression group. In addition, miR-200a-3p predicted NSCLC adverse prognosis with an AUC of 0.653, a sensitivity of $64.52 \%$ and a specificity of $68.97 \%$. GATA6 predicted NSCLC adverse prognosis with an AUC of 0.616 , a sensitivity of $48.39 \%$ and a specificity of $75.86 \%$, indicating that high expression of miR-200a-3p and low expression of GATA6 predicted poor prognosis. Furthermore, after adjusting for TNM stage, lymph node metastasis, distant metastasis, GATA6, CEA, NSE and SCCAg in the logistic regression model, high miR-200a-3p expression was indicated to increase the risk of death in patients with NSCLC, indicating that miR-200a-3p expression was independently associated with the poor prognosis of NSCLC. $\mathrm{miR}-200 \mathrm{a} / \mathrm{b} / \mathrm{c}$ are potential prognostic indicators of LUSC (40). GATA6 is decreased in high-grade LUAD $(50,51)$, and its reduction can enhance the metastatic potency in LUAD (20). In summary, high expression of miR-200a-3p and low expression of GATA6 predicted poor prognosis in patients with NSCLC.

In conclusion, high expression of miR-200a-3p and low expression of GATA6 in the peripheral blood were associated with clinical features and serum markers, and predicted poor prognosis in patients with NSCLC. Restoration of GATA6 expression or inhibition of miR-200a-3p may therefore represent a possibility for NSCLC early treatment. This was a case-control study, which revealed the clinical diagnostic efficacy of miR-200a-3p and GATA6 in NSCLC. However, there are still certain limitations in the present study: i) The 
sample size was small and the diagnostic and prognostic value of miR-200a-3p and GATA6 still requires further verification in a cohort with an appropriate sample size; ii) serum and plasma miRNAs mainly come from exosomes: Because of their increased number when diseases invade the body, stable existence in body fluids, easy access and resistance to enzymatic hydrolysis, they are considered to be ideal biomarkers for early cancer diagnosis. Serum exosomal miRNAs not only can be used for the early diagnosis of lung cancer, but also have the potential to distinguish NSCLC and SCLC (52). Whether the level of miR-200a-3p in the peripheral blood of patients with NSCLC included in the present study is associated with the level of serum exosomal miR-200a-3p, and whether the level of serum exosomal miR-200a-3p has the value of early diagnostic and prognostic evaluation of NSCLC still needs to be further studied; iii) the expression of miR-200a-3p and GATA6 in tumor samples were not detected; iv) the downstream targets of miR-200a-3p and GATA6 are not fully understood, and the regulatory mechanism of NSCLC still requires to be further elucidated. In the future, the regulatory upstream or downstream mechanism of miR-200a-3p and GATA6 in NSCLC will be further investigated, and in-depth functional research on miR-200a-3p and GATA6 will be conducted. Furthermore, the present study demonstrated that the expression of miR-200a-3p and GATA6 may be used for the early diagnosis and treatment of NSCLC, indicating that it may be associated with the intensity of tumor load in patients with NSCLC. Whether the postoperative expression of miR-200a-3p and GATA6 is also associated with the tumor load intensity remains to be explored. Detection of their expression following patient operation will help to evaluate the effect of radical operation and provide reference for further treatment after the operation.

\section{Acknowledgements}

Not applicable.

\section{Funding}

No funding was received.

\section{Availability of data and materials}

The datasets used and/or analyzed during the current study are available from the corresponding author on reasonable request.

\section{Authors' contributions}

JY, XYH and YBX contributed to the study concept and study design. CJF, HXW and LH contributed to the literature search. CJF and HXW contributed to manuscript preparation and YBX contributed to manuscript editing and review. JY, XYH, YBX and LH contributed to performing the experiments and data acquisition. CJF, HXW and LH contributed to data analysis and statistical analysis. JY and XYH confirm the authenticity of all the raw data. All authors have read and approved the final manuscript.

\section{Ethics approval and consent to participate}

The current study was approved by the Ethics Committee of Guizhou Provincial People's Hospital (approval no. LLS-2019123012; Guiyang, China). Written informed consent was obtained from each subject.

\section{Patient consent for publication}

Not applicable.

\section{Competing interests}

The authors declare that they have no competing interests.

\section{References}

1. Mukherjee TK, Malik P and Hoidal JR: The emerging role of estrogen related receptor $\alpha$ in complications of non-small cell lung cancers. Oncol Lett 21: 258, 2021.

2. Min HY and Lee HY: Mechanisms of resistance to chemotherapy in non-small cell lung cancer. Arch Pharm Res 44: 146-164, 2021.

3. Postmus PE, Kerr KM, Oudkerk M, Senan S, Waller DA, Vansteenkiste J, Escriu C and Peters S; ESMO Guidelines Committee: Early and locally advanced non-small-cell lung cancer (NSCLC): ESMO clinical practice guidelines for diagnosis, treatment and follow-up. Ann Oncol 28 (Suppl 4): iv1-iv21, 2017.

4. Alcibar OL, Nadal E, Romero Palomar I and Navarro-Martin A: Systematic review of stereotactic body radiotherapy in stage III non-small cell lung cancer. Transl Lung Cancer Res 10: 529-538, 2021.

5. D'Aniello C, Berretta M, Cavaliere C, Rossetti S, Facchini BA, Iovane G, Mollo G, Capasso M, Pepa CD, Pesce L, et al: Biomarkers of prognosis and efficacy of anti-angiogenic therapy in metastatic clear cell renal cancer. Front Oncol 9: $1400,2019$.

6. Dall'Olio FG, Abbati F, Facchinetti F, Massucci M, Melotti B, Squadrilli A, Buti S, Formica F, Tiseo M and Ardizzoni A: CEA and CYFRA 21-1 as prognostic biomarker and as a tool for treatment monitoring in advanced NSCLC treated with immune checkpoint inhibitors. Ther Adv Med Oncol 12: $1758835920952994,2020$.

7. Hatzakis KD, Froudarakis ME, Bouros D, Tzanakis N, Karkavitsas N and Siafakas NM: Prognostic value of serum tumor markers in patients with lung cancer. Respiration 69: 25-29, 2002.

8. Wu H, Wang Q, Liu Q, Zhang Q, Huang Q and Yu Z: The serum tumor markers in combination for clinical diagnosis of lung cancer. Clin Lab 66, 2020.

9. Popper HH, Ryska A, Tímár J and Olszewski W: Molecular testing in lung cancer in the era of precision medicine. Transl Lung Cancer Res 3: 291-300, 2014.

10. Guo Y, Cao R, Zhang X, Huang L, Sun L, Zhao J, Ma J and Han C: Recent progress in rare oncogenic drivers and targeted therapy for non-small cell lung cancer. Onco Targets Ther 12: 10343-10360, 2019.

11. Alzofon $\mathrm{N}$ and Jimeno A: Capmatinib for non-small cell lung cancer. Drugs Today (Barc) 57: 17-25, 2021.

12. Li LJ, Chang WM and Hsiao M: Aberrant expression of microrna clusters in head and neck cancer development and progression: Current and future translational impacts. Pharmaceuticals (Basel) 14: 194, 2021.

13. Papanota AM, Karousi P, Kontos CK, Ntanasis-Stathopoulos I, Scorilas A and Terpos E: Multiple myeloma bone disease: Implication of MicroRNAs in its molecular background. Int J Mol Sci 22: 2375, 2021.

14. Hammouz RY, Kołat D, Kałuzińska Ż, Płuciennik E and Bednarek AK: MicroRNAs: Their role in metastasis, angiogenesis, and the potential for biomarker utility in bladder carcinomas. Cancers (Basel) 13: 891, 2021.

15. Raue R, Frank AC, Syed SN and Brüne B: Therapeutic targeting of MicroRNAs in the tumor microenvironment. Int J Mol Sci 22: $2210,2021$. 
16. Liu C, Hu W, Li LL, Wang YX, Zhou Q, Zhang F, Song-Yang YY, Zhu W, Sun CC and Li DJ: Roles of miR-200 family members in lung cancer: More than tumor suppressors. Future Oncol 14: 2875-2886, 2018.

17. Xie K, Wang C, Qin N, Yang J, Zhu M, Dai J, Jin G, Shen H, $\mathrm{Ma} \mathrm{H}$ and $\mathrm{Hu} \mathrm{Z}$ : Genetic variants in regulatory regions of microRNAs are associated with lung cancer risk. Oncotarget 7: 47966-47974, 2016.

18. Wei S, Wang K, Huang $X$, Zhao Z and Zhao Z: LncRNA MALAT1 contributes to non-small cell lung cancer progression via modulating miR-200a-3p/programmed death-ligand 1 axis Int J Immunopathol Pharmacol 33: 2058738419859699, 2019.

19. Li H, Feng $\mathrm{C}$ and Shi S: miR-196b promotes lung cancer cell migration and invasion through the targeting of GATA6. Oncol Lett 16: 247-252, 2018.

20. Cheung WK, Zhao M, Liu Z, Stevens LE, Cao PD, Fang JE, Westbrook TF and Nguyen DX: Control of alveolar differentiation by the lineage transcription factors GATA6 and HOPX inhibits lung adenocarcinoma metastasis. Cancer Cell 23: 725-738, 2013.

21. Zang Q, Xu L, Li J and Jia H: GATA6 activated long non-coding RNA PCAT1 maintains stemness of non-small cell lung cancer by mediating FRK. J BUON 25: 2371-2381, 2020.

22. Dong H, Weng C, Bai R, Sheng J, Gao X, Li L and Xu Z: The regulatory network of miR-141 in the inhibition of angiogenesis. Angiogenesis 22: 251-262, 2019.

23. Travis WD, Brambilla E, Nicholson AG, Yatabe Y, Austin JHM, Beasley MB, Chirieac LR, Dacic S, Duhig E, Flieder DB, et al: The 2015 World Health Organization classification of lung tumors: Impact of genetic, clinical and radiologic advances since the 2004 classification. J Thorac Oncol 10: 1243-1260, 2015.

24. Ren Y, Qiu H, Yuan Y, Ye J, Tian Y, Wen B, Zhang W and Li Q Evaluation of 7th edition of AJCC staging system for nasopharyngeal carcinoma. J Cancer 8: 1665-1672, 2017.

25. Cheng Y, Qu X, Dong Z, Zeng Q, Ma X, Jia Y, Li R, Jiang X, Williams C, Wang T and Xia W: Comparison of serum exosome isolation methods on co-precipitated free microRNAs. PeerJ 8 : e9434, 2020.

26. Madadi S and Soleimani M: Comparison of miR-16 and cel-miR-39 as reference controls for serum miRNA normalization in colorectal cancer. J Cell Biochem 120: 4802-4803, 2019.

27. Mitchell PS, Parkin RK, Kroh EM, Fritz BR, Wyman SK, Pogosova-Agadjanyan EL, Peterson A, Noteboom J, O'Briant KC, Allen A, et al: Circulating microRNAs as stable blood-based markers for cancer detection. Proc Natl Acad Sci USA 105: 10513-10518, 2008.

28. Lee H, Kim C, Kang H, Tak H, Ahn S, Yoon SK, Kuh HJ, Kim W and Lee EK: microRNA-200a-3p increases 5-fluorouracil resistance by regulating dual specificity phosphatase 6 expression. Exp Mol Med 49: e327, 2017.

29. Livak KJ and Schmittgen TD: Analysis of relative gene expression data using real-time quantitative PCR and the 2(-Delta Delta C(T)) method. Methods 25: 402-408, 2001.

30. Campbell JD, Alexandrov A, Kim J, Wala J, Berger AH, Pedamallu CS, Shukla SA, Guo G, Brooks AN, Murray BA, et al: Distinct patterns of somatic genome alterations in lung adenocarcinomas and squamous cell carcinomas. Nat Genet 48: 607-616, 2016.

31. Cancer Genome Atlas Research Network: Comprehensive genomic characterization of squamous cell lung cancers. Nature 489: 519-525, 2012

32. Cancer Genome Atlas Research Network: Comprehensive molecular profiling of lung adenocarcinoma. Nature 511: 543-550, 2014.

33. Zhang J, Zhu $\mathrm{N}$ and Chen $\mathrm{X}$ : A novel long noncoding RNA LINC01133 is upregulated in lung squamous cell cancer and predicts survival. Tumour Biol 36: 7465-7471, 2015.

34. Zhong S, Golpon H, Zardo P and Borlak J: miRNAs in lung cancer. A systematic review identifies predictive and prognostic miRNA candidates for precision medicine in lung cancer. Transl Res 230: 164-196, 2021

35. Peng L, Wang Y, Liu F, Qiu X, Zhang X, Fang C, Qian X and $\mathrm{Li} \mathrm{Y}$ : Peripheral blood markers predictive of outcome and immune-related adverse events in advanced non-small cell lung cancer treated with PD-1 inhibitors. Cancer Immunol Immunother 69: 1813-1822, 2020.
36. Tanizaki J, Haratani K, Hayashi H, Chiba Y, Nakamura Y, Yonesaka K, Kudo K, Kaneda H, Hasegawa Y, Tanaka K, et al: Peripheral blood biomarkers associated with clinical outcome in non-small cell lung cancer patients treated with nivolumab. J Thorac Oncol 13: 97-105, 2018.

37. Weidle UH, Birzele F and Nopora A: MicroRNAs as potential targets for therapeutic intervention with metastasis of non-small cell lung cancer. Cancer Genomics Proteomics 16: 99-119, 2019.

38. Chen Y, Peng W, Lu Y, Chen J, Zhu YY and Xi T: MiR-200a enhances the migrations of A549 and SK-MES-1 cells by regulating the expression of TSPAN1. J Biosci 38: 523-532, 2013.

39. He Q, Fang Y, Lu F, Pan J, Wang L, Gong W, Fei F, Cui J, Zhong J, Hu R, et al: Analysis of differential expression profile of miRNA in peripheral blood of patients with lung cancer. J Clin Lab Anal 33: e23003, 2019.

40. Shen Y, Pan X and Yang J: Gene regulation and prognostic indicators of lung squamous cell carcinoma: TCGA-derived miRNA/mRNA sequencing and DNA methylation data. J Cell Physiol 234: 22896-22910, 2019.

41. Chen W, Chen Z, Zhang M, Tian Y, Liu L, Lan R, Zeng G, Fu X, $\mathrm{Ru} \mathrm{G}$, Liu W, et al: GATA6 exerts potent lung cancer suppressive function by inducing cell senescence. Front Oncol 10: 824, 2020.

42. Fumagalli C, Bianchi F, Raviele PR, Vacirca D, Bertalot G, Rampinelli C, Lazzeroni M, Bonanni B, Veronesi G, Fusco N, et al: Circulating and tissue biomarkers in early-stage non-small cell lung cancer. Ecancermedicalscience 11: 717, 2017.

43. Halvorsen AR, Bjaanæs M, LeBlanc M, Holm AM, Bolstad N, Rubio L, Peñalver JC, Cervera J, Mojarrieta JC, López-Guerrero JA, et al: A unique set of 6 circulating microRNAs for early detection of non-small cell lung cancer. Oncotarget 7: 37250-37259, 2016.

44. Wang C, Ding M, Xia M, Chen S, Van Le A, Soto-Gil R, Shen Y, Wang N, Wang J, Gu W, et al: A Five-miRNA panel identified from a multicentric case-control study serves as a novel diagnostic tool for ethnically diverse non-small-cell lung cancer patients. EBioMedicine 2: 1377-1385, 2015.

45. Fan X, Chen X, Feng Q, Peng K, Wu Q, Passerini AG, Simon SI and Sun C: Downregulation of GATA6 in mTOR-inhibited human aortic endothelial cells: Effects on TNF- $\alpha$-induced VCAM-1 expression and monocytic cell adhesion. Am J Physiol Heart Circ Physiol 316: H408-H420, 2019.

46. Si L, Tian H, Yue W, Li L, Li S, Gao C and Qi L: Potential use of microRNA-200c as a prognostic marker in non-small cell lung cancer. Oncol Lett 14: 4325-4330, 2017

47. Mongroo PS and Rustgi AK: The role of the miR-200 family in epithelial-mesenchymal transition. Cancer Biol Ther 10: 219-222, 2010.

48. Xu-Welliver $\mathrm{M}$ and Carbone DP: Blood-based biomarkers in lung cancer: Prognosis and treatment decisions. Transl Lung Cancer Res 6: 708-712, 2017

49. Sun X, Wang M, Xu R, Zhang D, Liu A, Wang Y, Lu T, Xin Y, Zhao Y, Xuan Y, et al: Prognostic model based on circular RNA circPDK1 for resected lung squamous cell carcinoma. Transl Lung Cancer Res 8: 907-919, 2019.

50. Hu H, Sun Z, Li Y, Zhang Y, Li H, Zhang Y, Pan Y, Shen L, Wang R, Sun Y and Chen H: The histologic classifications of lung adenocarcinomas are discriminable by unique lineage backgrounds. J Thorac Oncol 11: 2161-2172, 2016.

51. Nakajima N, Yoshizawa A, Nakajima T, Hirata M, Furuhata A, Sumiyoshi S, Rokutan-Kurata M, Sonobe M, Menju T, Miyamoto E, et al: GATA6-positive lung adenocarcinomas are associated with invasive mucinous adenocarcinoma morphology, hepatocyte nuclear factor $4 \alpha$ expression, and KRAS mutations. Histopathology 73: 38-48, 2018.

52. Masaoutis C, Mihailidou C, Tsourouflis G and Theocharis $S$ Exosomes in lung cancer diagnosis and treatment. From the translating research into future clinical practice. Biochimie 151: 27-36, 2018.

This work is licensed under a Creative Commons Attribution-NonCommercial-NoDerivatives 4.0 International (CC BY-NC-ND 4.0) License. 